\title{
A close look into an intermediate redshift galaxy using STIS
}

\author{
D. F. de Mello ${ }^{1}$ and A. Pasquali ${ }^{2}$
}

\begin{abstract}
1 Onsala Space Observatory, Chalmers University of Technology, 43992 Onsala, Sweden
2 ESO/ST-ECF, Karl-Schwarzschild-Strasse 2, 85748 Garching bei Muenchen, Germany e-mail: apasqual@eso.org
\end{abstract}

Received 6 June 2001 / Accepted 26 August 2001

\begin{abstract}
We present a detailed view of a galaxy at $z=0.4$ which is part of a large database of intermediate redshifts using high resolution images. We used the STIS parallel images and spectra to identify the object and obtain the redshift. The high resolution STIS image $\left(0.05^{\prime \prime}\right)$ enabled us to analyse the internal structures of this galaxy. A bar along the major axis and hot-spots of star formation separated by $0.37^{\prime \prime}(1.6 \mathrm{kpc})$ are found along the inner region of the galaxy. The analysis of the morphology of faint galaxies like this one is an important step towards estimating the epoch of formation of the Hubble classification sequence.
\end{abstract}

Key words. faint galaxies - barred galaxies - mergers - galaxy evolution

\section{Introduction}

The attempts to connect the luminosity density in highredshift galaxy samples to lower-redshift surveys (e.g. Madau et al. 1996; Steidel et al. 1999) suggest that star formation density increases rapidly from $z=0$ to $z=1$. However, despite the efforts made so far, the nature of the objects and the mechanisms which are the drivers of galaxy evolution at intermediate redshifts are still under debate. The strong clustering of faint galaxies at intermediate redshifts (Infante et al. 1995; Carlberg et al. 2001) suggests that mergers were more frequent at that time than in the present and would be responsible for an increase of the star formation at those epochs. It is also possible that there is an excess of dwarf galaxies having their first burst of star formation at intermediate redshifts (Babul \& Ferguson 1996; Guzmán 1998). Therefore, a census of the galaxy population as a function of redshift would be ideal in order to better understand the nature of galaxies at different epochs. The analysis of the morphology of faint galaxies is a key step towards estimating the epoch of formation of the Hubble classification sequence. Deep exposures such as the Hubble Deep Field (HDF) campaigns (Williams et al. 1996; Williams et al. 2000) have enabled morphology classification of faint galaxies for the first time (e.g. van den Bergh et al. 1996). However, larger samples are required in order to draw an unbiased view of galaxy evolution. For instance, in the sample of Abraham et al. (1999), which includes the HDF and the HDF-South there are only 15 spiral systems at $z<0.5$. Our aim is to improve this situation by assembling a statistically large

Send offprint requests to: D. F. de Mello, e-mail: duilia@oso.chalmers.se database of galaxies at intermediate redshifts using high resolution images. In this letter we present the first field we have analysed.

\section{The data - why STIS?}

We are selecting our sample from the HST Space Telescope Imaging Spectrograph (STIS) high latitude fields, which have been observed in parallel mode. STIS is used in parallel with any other HST instrument selected as prime instrument for a specific program (GO). Therefore, STIS points to a field adjacent to the GO and it is programmed to acquire direct images (in the CLEAR Filter covering from $2000 \AA$ to $1 \mu \mathrm{m}$ ) and slitless spectra (with the G750L grating) of the parallel field, with exposure times which fit the GO integrations. Direct images are characterized by a spatial sampling of $0.05^{\prime \prime}$, the highest offered by HST, and cover a field of view of $51^{\prime \prime} \times 51^{\prime \prime}$. They are used to derive the morphology of the galaxies in the field and to analyse their environments. The STIS slitless spectra of the same field (with a spectral resolution of $4.9 \AA /$ pix) over the spectral range 5230-10270 $\AA$ are extracted and searched for either emission lines or Balmer jump which will define the redshift of the galaxies in the parallel field.

The data presented in this letter were acquired with the HST/STIS spectrograph in parallel mode (Programme $\mathrm{ID}=8549$, PI: Baum) for a field at $\mathrm{RA}(2000)=$ $13^{\mathrm{h}} 37^{\mathrm{m}} 56^{\mathrm{s}} .9$ and $\operatorname{DEC}(2000)=70^{\circ} 16^{\prime} 54^{\prime \prime} .8$. Two pairs of direct and slitless-spectra images were taken in February 2000; the individual fits files are summarized in Table 1 together with their exposure times. The primary instrument was WFPC2 dedicated to the imaging of the cluster Abell 2218 (Programme ID = 8500, PI: Fruchter). 
Table 1. Log of the observations.

\begin{tabular}{cc|cc}
\hline Images & $\begin{array}{c}\text { Exp. Time } \\
\mathrm{s}\end{array}$ & Spectra & $\begin{array}{c}\text { Exp. Time } \\
\mathrm{s}\end{array}$ \\
\hline o5lil0sgq & 642 & o5lil0siq & 900 \\
o5lil0slq & 150 & o5lil0smq & 1397 \\
\hline
\end{tabular}

Abell $2218(z=0.17)$ is at $\mathrm{RA}(2000)=16^{\mathrm{h}} 35^{\mathrm{m}}$ and $\operatorname{DEC}(2000)=66^{\circ} 12^{\prime}$ (Smail et al. 2001) and, therefore, not included in the parallel images which are $\sim 3$ hours to the west and 4 degrees to the north of Abell 2218.

The direct images presented here were acquired in the MIRVIS configuration, with the 50CCD aperture and the CLEAR filter (Fig. 1). A number of pointings adjacent to the field are available in the archive, but no overlap between them was found.

The raw data were processed with the standard STIS pipeline using the best reference files. Subsequently, the images were aligned and added up with the STSDAS/CRREJ routine which allows for cosmic-rays cleaning. A final exposure of $792 \mathrm{~s}$ was obtained for the field direct imaging (Fig. 1). The spectra were taken with the same 50CCD aperture (i.e. no slit was used) and the G750L grating whose central wavelength is $7751 \AA$. In this set-up, the G750L grating is characterised by a spectral resolution of $\sim 5 \AA$ per pixel. The STECF/SLITLESS routine was used to locate the spectra of the brighter objects in the field in both the slitless-spectra images. The spectra of three galaxies (A, B, and C) and their adjacent backgrounds were extracted from each slitlessspectra image with a slit of $0.3^{\prime \prime}, 0.8^{\prime \prime}$ and $0.5^{\prime \prime}$ respectively. Following the background subtraction, each set of spectra was manually cleaned from cosmic rays and calibrated in wavelength by applying the dispersion correction: $\lambda=\left(\right.$ pixel - pixel $\left._{\mathrm{g}}\right) \times 4.9+7751$; where pixel $_{\mathrm{g}}$ is the $X$ coordinate of each galaxy in the corresponding direct image. Only at this point, the spectra of each galaxy were corrected for the grating response and added up, so that the final spectrum of each galaxy has a total exposure time of $2297 \mathrm{~s}$.

The spectra of galaxies B and C have low signal-tonoise to allow any unambiguous redshift determination. In the spectrum of the brightest object inside box A (hereafter, galaxy A) we identified an emission line at $9187 \AA$ as $\mathrm{H} \alpha$ which corresponds to $z=0.4$. Figures 2 and 3 show the rest frame spectrum where we have marked the position of the emission lines: [OIII]5007, [NII]6548, 6583 and [SII] 6716,6731 . However, only $\mathrm{H} \alpha$ and [OIII] $5007 \AA$ are $3 \sigma$ detections.

\section{Discussion}

The high resolution of STIS allows a detailed view of galaxy A, even though it is at a distance of $d_{\mathrm{A}}=885 \mathrm{Mpc}$ $\left(d_{\mathrm{L}}=1734 \mathrm{Mpc} ; H_{0}=75 \mathrm{~km} \mathrm{~s}^{-1} \mathrm{Mpc}^{-1}, q_{0}=0.5\right.$, $\left.\Omega_{\mathrm{m}}=1.0\right)$. The presence of a tail on the southern region and a disrupted "arm" on the east side are marked

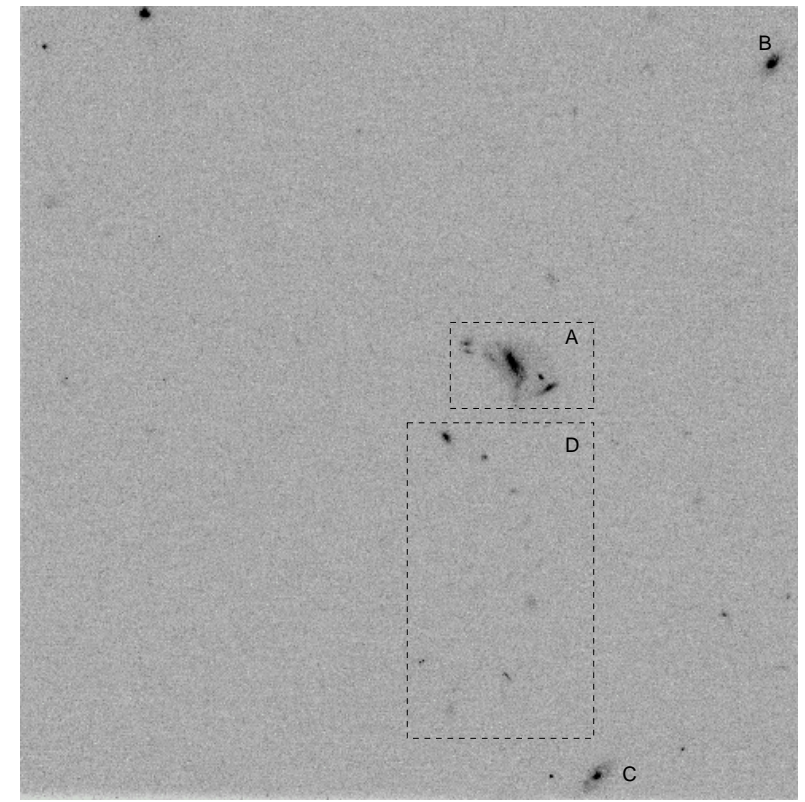

Fig. 1. STIS imaging of the field 204+70.2. Size of the field is $51^{\prime \prime} \times 51^{\prime \prime}$. Dashed box A is shown in detail in Fig. 4. North is to the top and East to left.

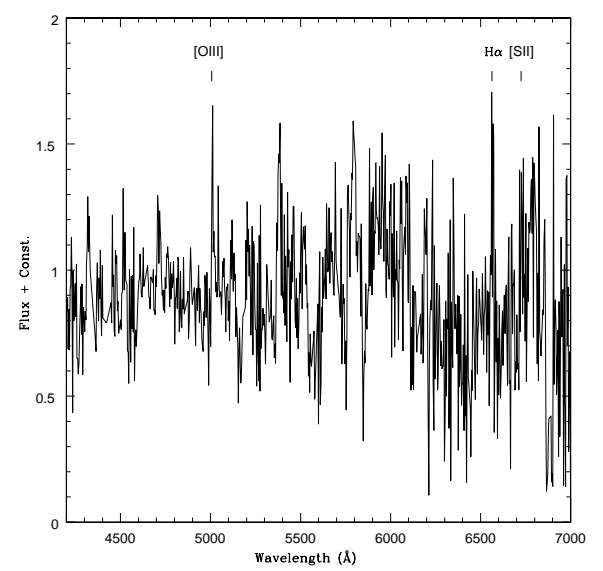

Fig. 2. Rest frame STIS spectrum of galaxy A in field $204+70.2$.

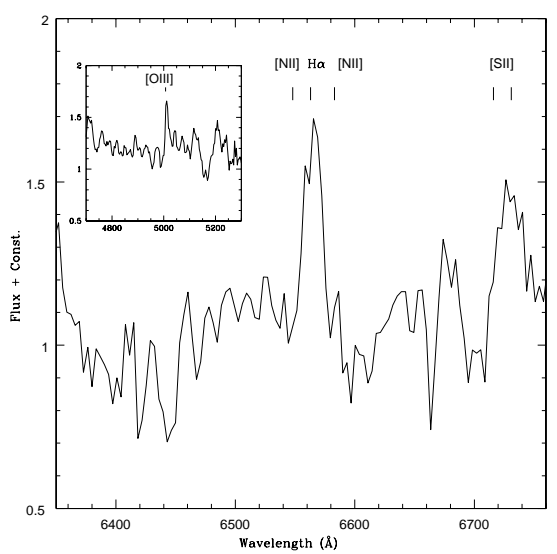

Fig. 3. Rest frame STIS spectrum of galaxy A in field $204+70.2$. Spectrum was smoothed with a boxcar of size 3 . [OIII]5007, [NII]6548, 6583 and [SII]6716, 6731 are marked. $\mathrm{H} \alpha$ and $[\mathrm{OIII}] 5007 \AA$ are $3 \sigma$ detections. 


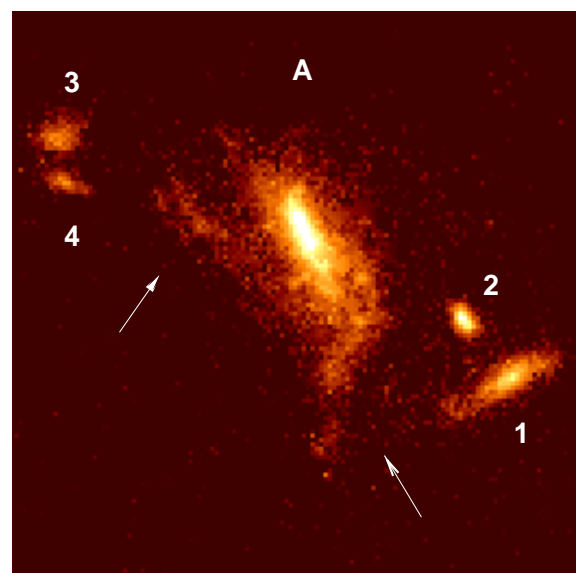

Fig. 4. Area A of field $204+70.2\left(6.5^{\prime \prime} \times 6.5^{\prime \prime}\right)$. Four faint galaxies are identified. A tail (south) and a disrupted arm (east) are marked with arrows. North is to the top and East is to left.

Table 2. Structural parameters of galaxy A.

\begin{tabular}{ccc}
\hline Semi-major axis $\left(^{\prime \prime}\right)$ & $\epsilon$ & PA $\left(^{\circ}\right)$ \\
\hline 1.0 & $0.67 \pm 0.02$ & $34.15 \pm 1.11$ \\
0.5 & $0.67 \pm 0.01$ & $27.72 \pm 0.83$ \\
0.1 & $0.64 \pm 0.07$ & $10.09 \pm 4.60$ \\
\hline
\end{tabular}

by arrows in Fig. 4. The symmetry of the morphology of galaxy A was analysed using contour maps (Figs. 5 and 6). The shape of the contours suggests the presence of a bar along the major axis. We used the IRAF/STSDAS task ELLIPSE to fit elliptical isophotes to the image (Fig. 7) and obtain the structural parameters, ellipticity and position angle ( $\epsilon$ and PA in Table 2). The change of $24^{\circ}$ in the orientation of the ellipses can be either due to the presence of a nuclear bar inclined with respect to the primary bar or to the presence of dust absorption and intense sites of star formation (Friedli 1996). The latter is probably the case in galaxy $\mathrm{A}$, since two opposite hot-spots separated by $0.37^{\prime \prime}\left(\sim 1.6 \mathrm{kpc} ; 1^{\prime \prime}=4.3 \mathrm{kpc}\right.$ at $\left.d_{\mathrm{A}}=885 \mathrm{Mpc}\right)$ are seeing in the internal region of the galaxy (Fig. 6).

To aid in the interpretation of the morphology of galaxy A we used the images generated by Hibbard \& Vacca $(1997)^{1}$ in their redshifting experiments of peculiar galaxies. We have binned the STIS image in order to have the same resolution as WFPC2. The contour maps produced show that the morphology of galaxy A is more symmetric and less extended than Arp 299 and NGC 1614. At $z=0.45$ Arp 299 is $9^{\prime \prime} \times 11^{\prime \prime}$ and NGC 1614 is $13^{\prime \prime} \times 11^{\prime \prime}$ whereas galaxy $\mathrm{A}$ is $2.75^{\prime \prime} \times 3.8^{\prime \prime}$. We have also compared galaxy A with NGC 1714 which is a member of Hickson Compact Group 31 and has other physical companions in a field of $12^{\prime \prime} \times 9^{\prime \prime}$. Although the group is significantly larger than field $\mathrm{A}$, the contours of the individual galaxies HCG31A (not including HCG31C) and HCG31B are

${ }^{1}$ Contour maps were produced using the same procedure as used for field A. Images were kindly provided by John Hibbard.

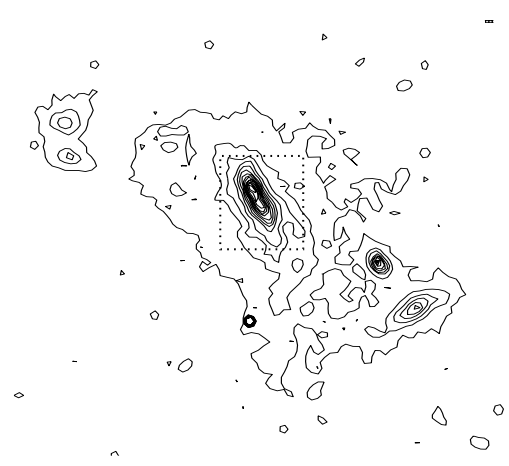

Fig. 5. Contour plot of area A of field $204+70.2$. The range of the contour levels and the number of contours are 53-500 and 12 , respectively. STIS background level is $37 \pm 8$ counts. North is to the top and East is to left.

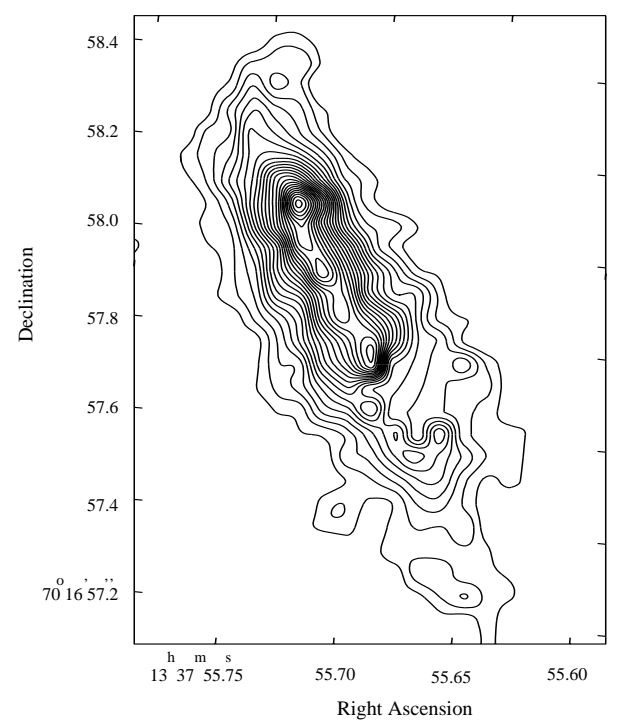

Fig. 6. Contour plot of internal region of galaxy A (diameter $\sim 5.6 \mathrm{kpc}$ ) - dashed box in Fig. 4. The range of the contour levels and the number of contours are 150-900 and 40, respectively. STIS background level is $37 \pm 8$ counts. North is to the top and East is to left.

similar to galaxy A showing symmetric internal contours and distorted outer contours.

There are four other small galaxies within field A (number 1 to 4 in Fig. 4); however, without complete redshift information no firm conclusion can be drawn regarding the environment of galaxy A and they could be chance alignments. If they are at $z=0.4$, galaxy number 1 has diameter $\sim 4.3 \mathrm{kpc}$ and the smallest, galaxy number 4 , has diameter $\sim 2.2 \mathrm{kpc}$. Their sizes are comparable to compact faint blue galaxies which have typical $R_{\mathrm{e}}<3 \mathrm{kpc}$ (Guzmán et al. 1997).

The CLEAR filter covers from $2000 \AA$ to $1 \mu \mathrm{m}$ which limits the astrophysical analysis that can be done with these data. However, we have estimated the magnitudes of our objects by comparing our fields with other images previously observed with HST. We have extracted a STIS parallel image which contains a region of the HDF. We have measured the aperture magnitudes of the HDF galaxies 
Table 3. CLEAR magnitudes (AB System).

\begin{tabular}{ccccc}
\hline Galaxy A & Galaxy 1 & Galaxy 2 & Galaxy 3 & Galaxy 4 \\
\hline 20.84 & 22.90 & 24.47 & 24.71 & 25.15 \\
\hline
\end{tabular}

with the CLEAR filter and compared these values with the cataloged HDF total magnitudes $\left(m_{\mathrm{t}}\right)^{2}$. After correction for background, the total counts are divided by the exposure time and multiplied by the inverse sensitivity $\left(8.9684505 \times 10^{-20} \mathrm{ergs} \mathrm{s}^{-1} \mathrm{~cm}^{-2} \AA^{-1}\right.$ counts $\left.^{-1}\right)$. The zero point of HST $(-21.10)$ was added. CLEAR magnitudes were found to be 1.053 magnitudes fainter than HDF magnitudes. CLEAR magnitudes (AB system) for galaxies in region A are given in Table 3. Galaxy A was recently observed at the Calar Alto $1.23 \mathrm{~m}$ telescope (F. Comeron private communication). $J, H$, and $K$ magnitudes were found to be $18.0 \pm 0.5,17.8 \pm 0.7$, and $18 \pm 0.4$.

We have also identified a string of 12 fainter galaxies on the south region of the STIS field (box D in Fig. 1). The peak-values of the counts in the faintest galaxies are between 3.3 and $37 \sigma$ detections. The faintest and the brightest galaxies in area D have CLEAR magnitudes 26.35 and 23.55 , respectively. The contour maps of 6 of these galaxies are typical of interacting spirals with disturbed morphologies. The other 6 galaxies are too faint to be morphologically classified.

\section{Conclusions}

In this letter we presented the first result of an ongoing project which aim at compiling a census of the galaxy population at intermediate redshifts. We are using STIS images taken in parallel mode to select our sample. The main advantage of the STIS images is the spatial resolution. Although, the exposure times are not long enough to reach the HDF faintest levels in magnitude, the high resolution enable us to see intermediate redshift galaxies in more detail. We presented a galaxy at $z=0.4$ in which a bar is clearly seen along its major axis. The presence of two opposite hot spots separated by $\sim 1.6 \mathrm{kpc}$ are seeing in the internal region of the galaxy. The presence of smaller galaxies in the same field is also reported. Followup observations of this field are planned in order to obtain complete redshift information.

Acknowledgements. We would like to thank N. Fourniol and the HST archive team at ST-ECF for their support in retrieving and processing the STIS data. We are grateful

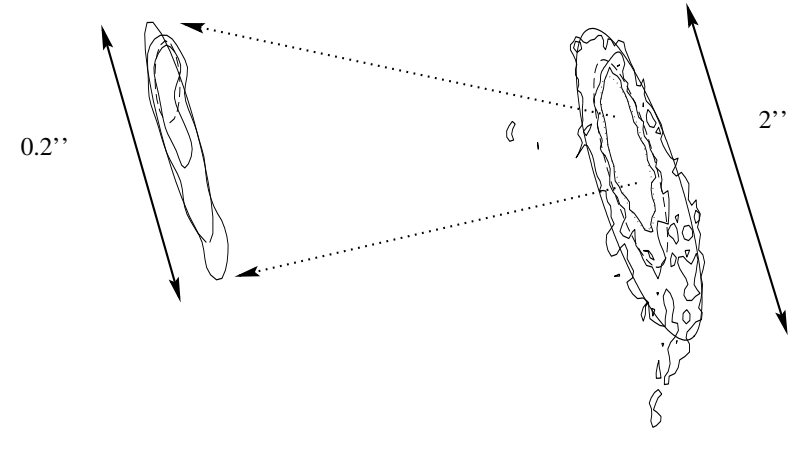

Fig. 7. Ellipses fitting the contour levels in the inner regions of galaxy A.

to our referee, M. Bershady, whose comments and criticisms helped improving our paper, to F. Schweizer and R. Carlberg for their inspiring comments and suggestions, and to J. Hibbard for making their images of peculiar galaxies available to us. This work was supported by the ESO Director General Discretionary Fund. D.F.M. is a "forskarassistent" of Vetenskapsrådet under project F620-489/2000.

\section{References}

Abraham, R. G., Merrifield, M. R., Ellis, R. S., et al. 1999, MNRAS, 308, 569

Babul, A., \& Ferguson, H. C. 1996, ApJ, 458, 1000

Carlberg, R. G., Yee, H. K. C., Morris, S. L., et al. 2001, ApJ, 552,427

Friedli, D. 1996, in Barred Galaxies, ed. R. Buta, D. A. Crocker, \& B. G. Elmegreen, ASP Conf. Ser., 91, 378

Guzmán, R. 1998, Ap\&SS, 263, 127

Guzmán, R., Gallego, J., Koo, D. C., et al. 1997, ApJ, 489, 559

Hibbard, J. E., \& Vacca, W. D. 1997, AJ, 114, 1741

Infante, L., de Mello, D. F., \& Menanteau, F. 1995, ApJ, 469, L85

Madau, P., Ferguson, H. C., Dickinson, M., et al. 1996, MNRAS, 283, 1388

Smail, I., Kuntschner, H., Kodama, T., et al. 2001, MNRAS, 323,839

Steidel, C. C., Adelberger, K. L., Giavalisco, M., et al. 1999, ApJ, 519, 1

van den Bergh, S., Abraham, R. G., Ellis, R. S., et al. 1996, AJ, 112, 359

Williams, R. E., Blacker, B., Dickinson, M., et al. 1996, AJ, 112, 1335

Williams, R. E., Baum S., Bergeron L. E., et al. 2000, AJ, 120, 2735

\footnotetext{
2 The HDF catalog used was taken from the HDF webaddress http://www.stsci.edu/ftp/science/hdf/archive/v2.html
} 